\title{
FISCALIDAD, DEMOGRAFÍA Y SOCIEDAD EN LA SONSIERRA EN TORNO A 1427: LAS FRONTERAS MERIDIONALES DEL REINO DE NAVARRA CON LA RIOJA CASTELLANA
}

\author{
Ernesto García Fernández \\ Universidad del País Vasco \\ Vitoria-Gasteiz
}

\section{Introducción*}

El Reino de Navarra durante la Edad Media tuvo incorporados una serie de territorios ubicados al Norte del Ebro y englobados a mediados del siglo XV, en algunos documentos de carácter fiscal, en una comarca conocida bajo el apelativo de Sonsierra. La recaudación del tributo de las medias primicias de la Merindad de Estella así lo certifica. En dicho documento aparecen incluidos en la región de la Sonsierra las villas y aldeas de San Vicente, Laguardia, Labraza y Viana'. Sonsierra, por tanto, era una expresión utilizada para designar a los habitantes que vivían al sur de la sierra que se extendía desde los montes de Toloño hasta más allá de la sierra de Codés. Ya desde mediados del siglo XII para describir esta comarca los monjes de San Millán de la Cogolla habían recurrido al vocablo latino "subserra", es decir, "debajo de la sierra", para referirse a quienes habitaban al oriente de la Sierra de Toloño, territorio este último Ilamado por entonces con el nombre de Tabuerneca².

* Este artículo se ha elaborado en el marco del proyecto de investigación financiado por el Ministerio de Ciencia e Innovación, Proyectos I+D 2008, titulado "Poder, sociedad y fiscalidad en las Merindades de Allende Ebro y La Rioja durante el Reinado de la dinastía Trastámara", cuya referencia es HAR2008-05841-C02-02. Proyecto integrado en la Red Temática Interuniversitaria Arca Comunis. Página web http://arcacomunis.uma.es/index.php.

1. GARCÍA FERNÁNDEZ, E., "La vida social, económica y religiosa de la Rioja Alavesa en la Baja Edad Media", Actas de las Primeras Jornadas de Estudios Históricos de la Rioja Alavesa. Espacio. Sociedad y Economía. Vitoria, 2002, pp. 135-158.

2. GARCÍA FERNÁNDEZ, E., "Tabuérniga: Reflexiones e hipótesis sobre una "civitate" altomedieval alavesa", Espacio, Tiempo y Forma, Madrid, (1992), pp. 13-38. 
En la Edad Moderna el vocablo Rioja se extendió a otras zonas, por motivos relacionados con la compraventa del vino y la competencia entre los productores del norte y sur del río Ebro. De este modo los vinos de las comarcas de San Vicente de la Sonsierra, Laguardia, Labraza y Viana comenzaron a ser considerados de Rioja. Hoy en día estas zonas, desde el punto de vista vitivinícola, forman parte de la Denominación de Origen Rioja, pero dicho nombre provino de zonas ubicadas al sur de la gran arteria acuosa que representa el río Ebro en la Península Ibérica. La Rioja estrictamente fue un territorio, en la Edad Media, que comprendió las tierras que rodeaban el Río Oja. Incluso existió en la Edad Media un distrito fiscal con dicho nombre, diferenciado de otras comarcas en la actualidad riojanas, pues no incluía las zonas integradas en la Merindad de Logroño, con una demarcación tributaria específica ${ }^{3}$. Esta circunstancia ya tenía su reflejo en la organización eclesiástica. Al menos desde mediados del siglo XIII se distinguían en la diócesis de Calahorra los arcedianatos de Logroño, de Nájera y de la Rioja. Por tanto, el vocablo Rioja se restringía a un marco espacial muy determinado ${ }^{4}$.

En consecuencia durante la Edad Media la denominación Rioja no fue un topónimo sociogeográfico con el que se refirieran las administraciones monárquicas castellanas y navarras a las comarcas referenciadas al norte del Ebro. Si fue habitual el uso de los términos "subserra" en el XII y "Sonsierra" en el XV para dichos territorios, sin embargo, San Vicente de la Sonsierra, será la única población que conservó la palabra Sonsierra como una parte consubstancial de su identidad. Quizá para diferenciarse de otras localidades que portaban el mismo nombre. Los demás pueblos de la Sonsierra no incorporaron dicho apelativo a su denominación particular.

Durante algunos años toda la comarca objeto de atención en esta investigación fue bautizada con el nombre de Laguardia, con el beneplácito de los reyes de Navarra. En 1164, el monarca Sancho VI de Navarra, dio fuero a la villa de Laguardia, cuya jurisdicción se extendió desde Buradón hasta el soto de Iñigo Galindo en términos de Viana, convirtiéndola en la capital administrativa de un territorio que comprendía asimismo las localidades de San Vicente de la Sonsierra y sus aldeas, Labraza y al menos parte de los futuros términos de Viana. El interés de los reyes navarros por reforzar sus fronteras frente a Castilla supu-

3. LADERO QUESADA, M. Á., La Hacienda Real castellana entre 1480 y 1492. Universidad de Valladolid, 1967.

4. Quiero señalar que en el plano eclesiástico estos territorios dependieron de los arcedianatos de Nájera (San Vicente de la Sonsierra y la mayoría de los términos jurisdiccionales de Laguardia, cuyo arciprestazgo incluía a la primera localidad y sus respectivas aldeas) y de Berberigo (Viana y Labraza). Véanse los trabajos de DíAZ BODEGAS, P., La diócesis de Calahorra y La Calzada en el siglo XIII (La sede, sus obispos e instituciones). Logroño, 1995 y GARCíA FERNÁNDEZ, E., "Iglesia, religiosidad y sociedad en el País Vasco durante el siglo XIV", Edad Media. Revista de Historia (Universidad de Valladolid), Volumen 8, pp. 99-144. 
so la potenciación de la creación de nuevas villas, que de esta manera se desgajaron de la jurisdicción de Laguardia. El proceso urbanizador se inició con la fundación de las villas de San Vicente de la Sonsierra en 1172, de San Cristóbal de Labraza en 1196 y de Viana en 1219. Las dos últimas villas fueron en última instancia obra de Sancho VII de Navarra, en cuyo reinado la "Álava nuclear", Guipúzcoa y el Duranguesado acabaron siendo arrebatados a Navarra por Alfonso VIII de Castilla. Por consiguiente San Vicente de la Sonsierra y sus aldeas pertenecieron a Laguardia durante 8 años. De igual modo Labraza y parte de Viana se mantuvieron en la jurisdicción de Laguardia a lo largo de 32 y 55 años respectivamente.

Por otra parte, los reyes castellanos pusieron en práctica una política urbanizadora similar en las tierras fronterizas a Navarra que se hallaban bajo su dominio. Dieron fuero de población a las villas de Haro en 1187, de Navarrete en 1195 , de Briones en $1256^{5}$ y de Labastida en $1242^{6}$. Privilegiar a los pobladores de estas nuevas urbanizaciones era una medida con la que se buscaba asimismo el fortalecimiento social, institucional y militar de estas comarcas casteIlanas limítrofes con el Reino de Navarra.

Pues bien en este artículo analizaré los contenidos del censo fiscal navarro de 1427, en lo que concierne a las villas y aldeas de San Vicente de la Sonsierra, Laguardia, Labraza y Viana, poblaciones navarras por aquellas fechas. Asimismo se publica en el apéndice documental la parte del "libro de fuegos" de la Merindad de Estella referente a la villa y aldeas de San Vicente de la Sonsierra. En 1427 la administración del reino de Navarra envío comisarios para recaudar los "cuarteles" a lo largo y ancho de los distintos lugares y villas distribuidos en las Merindades. Se conserva, entre otros, el censo elaborado en la Merindad de Estella, a cuya demarcación pertenecieron los pueblos y villas objeto de atención en este trabajo de investigación. En este censo había que anotar el número total de pobladores con casa y las cantidades a tributar por los pagadores considerados contribuyentes. El análisis comparativo de la distribución de la carga fiscal y del número de pobladores de las cuatro comarcas examinadas nos permitirá acercarnos a la estructura socioeconómica de aquellas gentes y territorios a principios del segundo cuarto del siglo XV.

5. MARTíNEZ DíEZ, G., "Fueros de la Rioja". Anuario de Historia del Derecho Español, Madrid, (1979), pp. 327-454.

6. GARCÍA FERNÁNDEZ, E., LÓPEZ LÓPEZ DE ULLIBARRI, F. y DÍAZ DE DURANA ORTIZ DE URBINA, J.R., Labastida en ladead Media: poblamiento y organización político-administrativa (s. X-XIII). Vitoria, 1990.

7. Logroño -esta localidad había sido fundada por Alfonso VI de Castilla en 1095-, Fuenmayor, Cenicero, Torremontalbo, Briones y Haro fueron poblaciones limítrofes con el Reino de Navarra. 


\section{Radiografía económica, fiscal, demográfica y social de las villas y aldeas de San Vicente, Laguardia, Labraza y Viana}

Los pobladores de estas cuatro circunscripciones jurídico-administrativas se dedicaron en el siglo $\mathrm{XV}$ de modo preferente al cultivo del cereal y de la vid. No descuidaron tampoco actividades ganaderas. De hecho, los puercos y los pequeños rebaños de ovejas formaron parte de sus ocupaciones profesionales. A este respecto es interesante describir las respuestas que dieron los vecinos a las preguntas formuladas por los comisarios en 1427. En unos casos las contestaciones fueron generales y en otros se acompañaron de matizaciones complementarias. El censo de 1427 es muy explícito al respecto.

Los moradores de San Vicente y sus aldeas juraron lo siguiente:

"Interrogados de que biven dixieron que la dicha billa et aldeas no an otra manera de bivir salvo que biben sobre la labranza de pan et bino e hun aynno con otro non cugen de pan para provision de la mitad del aynno et bino que cugen assaz para su provision et aun para bender enpero que del bino no se pueden aprovechar de que fue bedada la saqua del bino que non lo pueden bender sino a menoscabo de manera que casi no an provecho nin sostenimiento alguno".

De esta forma sostenían que sus provisiones de cereal eran escasas, mientras que su producción vinícola era considerable, pero que la política prohibicionista de exportar sus caldos a Castilla estaba teniendo efectos muy negativos para la economía de sus habitantes ${ }^{8}$.

Los pobladores de Laguardia y sus aldeas afirmaron que anualmente solían tener excedentes productivos de cereal y de vino, cuyas ventas permitían el sostenimiento de sus economías familiares:

"Interrogados de que biven dixieron que biven sobre la labrança de pan e bino et hun aynno con otro cugen pan et bino para su provisión e para bender et con aquello se sostienen sin que se ayuden nin husen de otros mantenimientos".

Los vecinos de Labraza y sus aldeas no sostuvieron de manera absoluta lo mismo, pues tuvieron en cuenta la existencia de familias cuya penuria les impe-

8. Es conocida la fuerte competencia económica existente por abastecer los mercados castellanos del norte -Vitoria y Salvatierra eran grandes centros consumidores de vino-, entre los productores de vino de la Rioja castellana y de la Sonsierra de Navarra. Los riojanos, como castellanos, se habían dirigido desde el siglo XIV a sus reyes solicitando el monopolio de la venta de dicho producto dentro del reino y exigiendo que los navarros -incluidos los moradores de la Sonsierra de Navarra- no pudieran exportar sus vinos tintos y blancos a Castilla. Los conflictos entre castellanos y navarros por esta cuestión desembocaron en momentos puntuales en graves incidentes durante los siglos XIV y XV. 
lía a vender su fuerza de trabajo en Viana (Navarra) o en Logroño (Castilla), labrando heredades ajenas:

"Interrogados que biven dixieron que biven sobre la labrança de pan e bino et que hun aynno con otro cugen pan e bino los medios de la billa para su provisión e los otros non para la mitat del aynno et que se sostienen sobre aquello et los mas alogandosse a labrar en Biana et en Logroyno et passan lo mejor que pueden su vida con grant trabajo".

Los pobladores de Viana y sus aldeas reprodujeron básicamente los argumentos expresados por los vecinos de San Vicente de la Sonsierra. Señalan, sin embargo, el uso de subterfugios varios para justificar la venta de una parte de la producción vinícola en Castilla, al tener que hacer algunos pagos a señores castellanos. Sin duda, se trataba de sortear las prohibiciones a la exportación de vino:

"Interrogados que manera tienen de vivir dixieron que biven sobre la labrança de pan e bino et que hun aynno con otro cugen pan et bino para su provisión et aun del bino para bender sino que non pueden bender el bino de que fue vedada la saqua sino que se remidan e den ciertas sumas de dineros a Lope de Rojas et a otras personas de Castilla e con todo non lo pueden bender sinon a grant menoscabo et con estos lo passan muy estrechamente et con los afruentos que an con los de Logroynno".

Por otra parte en el censo se ofrece información diversa referente a temas fiscales, a los privilegios de estas villas o a las condonaciones de parte de los tributos exigidos. Se suele relacionar estas exenciones con la situación fronteriza de estas poblaciones con Castilla. Así por ejemplo se anotaron las cuantías que cada territorio debía pagar en concepto de "cuartel" o de fonsadera, impuesto este último fijado en una cantidad a pagar por cada casa en los propios fueros otorgados por los reyes navarros.

Los vecinos de Labraza afirman tributar anualmente 20 libras de fonsadera, aunque señalan que la reina les había hecho gracia de dicho tributo. Al mismo tiempo justifican sus enormes gastos principalmente a causa de los desembolsos destinados a la fortificación de la villa y de su muralla. Para esta obra contrataron al constructor Martín Pérez de Laguardia, al que ya habían pagado 527 libras, 12 sueldos y 4 dineros. Igualmente afirman que habitualmente pagaban por "cuartel" 55 florines, cifra rebajada por el monarca navarro en un primer momento hasta 30 florines y en un segundo lugar hasta 20 florines. Asimismo adujeron que en la villa y aldeas se habían despoblado unas 35 casas, que representan el 125\% de las casas referenciadas en 1427 -ascienden a 28-.

Los vecinos de Viana y sus aldeas dijeron que regularmente pagaban cada año 61 sueldos fuertes en concepto de fonsadera y que solían tributar 249 flo- 
rines por "cuartel". Esta suma les fue reducida en una tercera parte por los reyes, debido a los gastos judiciales que tenían en particular con el concejo de Logroño y con algunos señores castellanos en relación con la veda de exportación de vino a Castilla. Las diferencias fiscales por "cuartel" entre Viana y Labraza son considerables, efecto en gran medida del menor número de pobladores y de la menor extensión territorial de la última localidad. En Viana el número de casas abandonadas fue muy elevado, 255 -un 123,78\%-, entre la villa, las aldeas existentes y las 5 desaparecidas.

Aldeas despobladas en la comunidad de Viana, según el censo fiscal de 1427

\begin{tabular}{|l|c|}
\hline \multicolumn{1}{|c|}{ NOMBRE DE LAS ALDEAS } & NÚMERO DE CASAS ABANDONADAS \\
\hline GARAYNO & - \\
\hline TIDON & - \\
\hline LONGAR & - \\
\hline PEREZUELAS & - \\
\hline PERAYTA & - \\
\hline TOTAL DE CASAS ABANDONADAS & 28 \\
\hline
\end{tabular}

En San Vicente y sus aldeas se afirma que pagaban 109 libras en concepto de fonsadera. También juraron las personas convocadas que solían contribuir anualmente con 105 florines por "cuartel", cantidad disminuida a 26 florines, gracias a las mercedes y remisiones recibidas de los reyes navarros. Pero además algunas aldeas, Peciña y Orzales, tenían diversas obligaciones de carácter señorial con Ruy López Dávalos y con el cenobio de San Salvador de Leire. La primera debía entregar la fonsadera a Ruy López Dávalos y la segunda debía pechar al monasterio navarro de San Salvador de Leire. En concreto todos los vecinos de Orzales, excepto uno, debían pagar al abad de Leire 4 robos de trigo y sendos de cebada por el solar en que vivían, lo que suponía 10 cahíces de trigo y 2,5 cahíces de cebada.

De otro lado los vecinos de Laguardia y sus aldeas afirmaron que entregaban habitualmente 16 libras al año de fonsadera y 435 florines por "cuartel", cantidad netamente superior a la exigida a la villa y aldeas de Viana. No hay constancia de reducción alguna por este capítulo tributario. Se recuerdan, empero, los numerosos gastos de la Comunidad, más de 100 florines al año, provenientes de los conflictos mantenidos con las tierras limítrofes de su jurisdicción: con Diego Pérez Sarmiento (Lagrán y Peñacerrada) y con los concejos de Logroño, Navarrete y Nájera.

Los procuradores de Laguardia y sus aldeas juraron ante los comisarios que a causa de la última guerra, de las mortandades y de movimientos migratorios 
se habían despoblado 676 casas $^{9}$. De ser cierto este dato no quedaría más remedio que aceptar un dramático descenso de la población: el 132\% de las casas de clérigos y legos cristianos francos enumeradas en el censo de 1427. Se trata de un tanto por ciento similar al expresado en sus respectivas comunidades por los procuradores de las villas y aldeas de Labraza (125\%), de Viana $(123,78 \%)$ y de San Vicente (118,97\%). Dar crédito a dichas noticias supondría que Laguardia y sus aldeas habrían alcanzado a fines del siglo XIV un número de 1.203 casas, sin contar judíos e hidalgos ${ }^{10}$, Labraza y sus aldeas 63 casas, Viana y sus aldeas 461 y San Vicente y sus aldeas 554 casas. La relativa coincidencia de los porcentajes de casas abandonadas en los cuatro centros jurídicoadministrativos estudiados pudiera obedecer a una realidad subyacente. Ahora bien, el análisis de los datos plantea algunas contradicciones.

Aldeas despobladas en la comunidad de Laguardia, según el censo fiscal de 1427

\begin{tabular}{|l|c|}
\hline \multicolumn{1}{|c|}{ NOMBRE DE LAS ALDEAS } & NÚMERO DE CASAS ABANDONADAS \\
\hline REINAVILLA & 20 \\
\hline ARMENTARANA & 13 \\
\hline MURRIARTE & 12 \\
\hline NAVARIDAS DE SUSO & 20 \\
\hline QUINTANILLA & 20 \\
\hline ESTOBLEDO & 12 \\
\hline PAZUENGOS & 10 \\
\hline TOTAL DE CASAS ABANDONADAS & 107 \\
\hline
\end{tabular}

Fijándome en el caso de Laguardia, salta a la vista que estas informaciones no casan en modo alguno con el censo de población de 1366, que incluyendo a hidalgos y judíos no sobrepasa los 600 hogares fiscales. Tampoco concuerda con el censo fiscal de 1350 que recoge 695 fuegos de población, dos años después de la Peste Negra de 1348. Eso sí, en 1350 no se anotaron ni judíos, ni

9. Sobre los períodos de mortandad remito al libro de MONTEANO SORBET, P. J., La ira de Dios. Los navarros en la Era de la Peste (1348-1723). Pamplona, 2002, que señala como especialmente agresivos los años 1348, 1362-1363, 1373-1374, 1382-1383, 1387, 1395, 1400 $1403,1411-1413,1422$ y 1423.

10. Se suman las casas supuestamente abandonadas en la villa y las aldeas pobladas, más las que existieron en las siete aldeas que se señalan como despobladas por aquellas fechas (Reinavilla, Armentarana, Murriarte, Navaridas de suso, Quintanilla, Estobledo y Pazuengos). Serían 107 el número de casas abandonadas en estas aldeas y 569 en la villa y aldeas -280 en la villa-. Sin embargo, Pazuengos, de la que se dice había contado con 10 casas, es anotada igualmente en el censo con dos pobladores en la relación de aldeas pobladas. 
hidalgos, lo que incrementaría quizá en más de 100 fuegos la cifra anterior ${ }^{11}$. La toma en consideración de 795 hogares para mediados del XIV significaría que este volumen poblacional estaba relativamente próximo a la equivalencia que los historiadores han hecho entre el montante del pago de la fonsadera y el número de casas existentes en la comarca unos años antes de 1348. La suma depositada por este concepto basculó entre 42 y 48 libras. Por cada casa se pagaban 12 dineros. Laguardia y sus aldeas contaban, por tanto, con un máximo de 950 casas antes de la "Peste Negra".

Hay historiadores que han afirmado que la "Peste Negra" causó en la Merindad de Estella una recesión demográfica superior al $60 \%$ de la población ${ }^{12}$. Si damos valor a los datos fiscales de la fonsadera de la Laguardia y sus aldeas la reducción supuso en esta zona el $20 \%$, porcentaje que podría aumentarse algo más si damos por sentada la existencia de fraudes voluntarios o involuntarios.

Por tanto, es posible que la "Peste Negra" hubiera incidido en la zona de Laguardia reduciendo cerca de un $30 \%$ sus efectivos demográficos. En consecuencia, antes de la desgraciada incidencia de esta epidemia, la población debió rondar las 1.150 casas, cifra que se aproxima, aunque está por debajo, a la suma de las casas habitadas en 1427 y de las supuestamente abandonadas desde fines del XIV (1.203, sin incluir a hidalgos y judíos).

En fin, las afirmaciones realizadas en 1427 bajo juramento por los procuradores de la Sonsierra hay que ponerlas en entredicho, si tan sólo se han de retrotraer los datos señalados hasta finales del siglo XIV, pero son dignas de ser tenidas en consideración si se contempla una memoria histórica que supera dicho marco cronológico. Es evidente, que son enormes las dificultades para resolver las interrogantes que sobrevienen del análisis de las fuentes documentales disponibles en la actualidad. Ello no ha sido óbice para que los investigadores se hayan acercado y analizado con detalle y minuciosidad unos textos tan ricos como los censos de carácter fiscal conservados en el Archivo General de Navarra ${ }^{13}$.

11. GARCíA FERNÁNDEZ, E., La Comunidad de Laguardia en la Baja Edad Media (13501516). Vitoria, 1985, pp. 63 y siguientes.

12. MARTÍN DUQUE, Á. J., "Vida urbana y vida rural en Navarra en el siglo XIV. Algunos materiales y sugerencias", en La sociedad vasca rural y urbana en el marco de la crisis de los siglos XIV y XV. Bilbao, edit. Diputación Foral de Vizcaya, 1975, en la página 47 afirma que la Merindad de Estella sufrió una pérdida del $63 \%$ de la población a consecuencia de la Peste Negra.

13. CARRASCO PÉREZ, J., La población de Navarra en el siglo XIV. Pamplona, 1973; BERT$\mathrm{HE}$, Maurice Famines et épidémies dans les campagnes navarraises á la fin du Moyen Âge. Paris, 1984, 2 vols.; RAMÍREZ VAQUERO, E., "Población, nobleza y renta señorial". Actas del Segundo Congreso Mundial Vasco. Vol. II, Vitoria-Gasteiz, 1988, pp. 298-312; ZABALO ZABALEGUI, J., "El reparto de las contribuciones extraordinarias. La 'ayuda' de Pamplona de 1366". Revista Príncipe de Viana, 193 (1992), pp. 429-441; GARCÍA FERNÁNDEZ, E., "Fiscalidad y sociedad

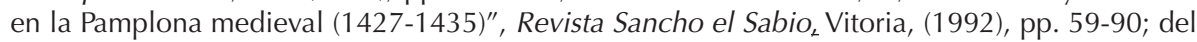
mismo autor "Fiscalidad y niveles de renta de la población de Estella a comienzos del siglo XV", 
Desde un punto de vista demográfico en el censo de 1427, tal como se ha comentado unas líneas antes, estuvieron ausentes los judíos -comunidad de importancia en las villas de Viana y Laguardia- y los hidalgos locales. Los datos ofrecidos por el censo que a continuación voy a examinar han de incrementarse con estos dos colectivos. Esta eventualidad demuestra que el impuesto de 1427 no fue de carácter universal, es decir, no afectó a todos los contribuyentes del reino asentados en estos territorios ribereños del Ebro ${ }^{14}$. Del censo se obtienen informaciones asimismo sobre aquellas familias con menos recursos económicos y de los "fuegos de población" femenina.

Fuegos de población de la Sonsierra de Navarra (año 1427)

\begin{tabular}{|l|c|c|c|c|}
\hline & $\begin{array}{c}\text { Laguardia } \\
\text { y sus 18 aldeas }\end{array}$ & $\begin{array}{c}\text { San Vicente } \\
\text { y sus 4 aldeas }\end{array}$ & $\begin{array}{c}\text { Viana } \\
\text { y sus 4 aldeas }\end{array}$ & $\begin{array}{c}\text { Labraza } \\
\text { y sus 2 aldeas }\end{array}$ \\
\hline Casas pobladas & 527 & 253 & 206 & 28 \\
\hline
\end{tabular}

En concreto, el número de "fuegos de población" de la comarca de la Sonsierra de Navarra asciende en 1427 a la cifra de 1.014. El 31\% del conjunto de los fuegos de la Merindad de Estella (3.266) 15 $^{15}$ Los 1.014 hogares o "fuegos" se distribuyen en la Sonsierra de Navarra de la siguiente manera: Laguardia y sus aldeas representan el 51,97\%, San Vicente y sus aldeas el 24,95\%, Labraza y sus aldeas tan sólo el 2,46\% y Viana y sus aldeas el 20,31\%. Teniendo en cuenta exclusivamente los pobladores que conformaban las distintas cabeceras político-administrativas los datos del censo de 1427 constatan que la villa de Laguardia contaba con el $31,11 \%$ de los hogares, mientras que en las aldeas estaban asentados el 68,88\%. En la villa de San Vicente por el contrario se censaban el $54,94 \%$ de las casas y en las aldeas se hallaban el 45,05\%. En la villa de Viana el número de hogares alcanzaba el 86,40\%, quedando tan sólo en las aldeas el 13,59\%. En la villa de Labraza este porcentaje se incrementaba hasta el $89,28 \%$ y el $10 \%$ restante en sus dos aldeas pobladas. En esas fechas las cua-

Historia, Instituciones y documentos, 21, Sevilla, (1994), pp. 345-366 y MONTEANO SORBET, P. J., "Navarra de 1366 a 1428: población y poblamiento". Revista príncipe de Viana, nº 208 (1995), pp. 307-343.

14. MONTEANO SORBET, P. J., Los navarros ante el hambre, la peste, la guerra y la fiscalidad. Pamplona, 1999, pp. 282-292.

15. MONTEANO SORBET, P. J., Los navarros ante el hambre, la peste, la guerra y la fiscalidad...etc., p. 330. Este historiador recoge en un cuadro el número de fuegos en Navarra en 1427-1429, según las circunscripciones para las que se disponen de datos, señalando 3.266 en la merindad de Estella que pagó 1.867 florines; 3.458 en la Merindad de Sangüesa, que contribuyó con 1.867 florines y 3.123 en la Merindad de las Montañas con Pamplona, que cotizó 1.128 florines. 
tro villas juntas concentraban casi el 50\% de las casas censadas en la Sonsierra de Navarra, pues se ubicaban en aquéllas el 49,90\%.

Tomando en consideración el número de casas abandonadas en las cuatro villas, se puede afirmar que la crisis demográfica y económica se había cebado de manera particular en estas cuatro localidades, pues habían desaparecido en torno al $62 \%$ de los hogares, frente al 38\% en las aldeas. Este dato, si es cierto, validaría la tremenda sangría demográfica acaecida en las villas de la Sonsierra, aunque este dramatismo se reduciría, al menos, en unos 6 puntos, hasta el 56\%, si valoramos las casas que existieron en las aldeas despobladas de Laguardia y $V_{i a n a}{ }^{16}$. Precisamente la suma de las casas habitadas y abandonadas de las villas da como resultado la existencia en otro tiempo de 444 fuegos de población en Laguardia, de 379 en San Vicente de la Sonsierra, de 349 en Viana y tal vez de unos 50 en Labraza. Las casas abandonadas en las villas respecto a las desaparecidas en las aldeas de sus respectivas jurisdicciones representan un $41 \%$ en la villa de Laguardia, un 79\% en la villa de San Vicente y un 75\% en la villa de Viana.

Pero no todos los vecinos pagaron el impuesto. Se enumeran las familias excusadas de pagar el tributo por carencia de bienes o debido a que fueron tipificados como pobres. La documentación engloba a estos últimos bajo la denominación de "impotentes". En la villa de Laguardia suponían el 42,97\% de las censados, mientras que en las aldeas se hallaban el $57,07 \%$ de los vecinos. En la villa de San Vicente se elevó dicho porcentaje hasta el 54,05\%, quedando las aldeas con el 45,94\%. En la villa de Labraza el 100\% de los "impotentes" residían dentro del recinto amurallado. No disponemos de datos para la villa de Viana, si bien en el conjunto de villa y aldeas alcanzaban el $22,33 \%$ de los habitantes de la jurisdicción. En toda la Sonsierra los "impotentes" representaron en 1427 el 20,71\% de la población. Es decir, cerca de una cuarta parte de los hogares se hallaba en unas condiciones de vida que podríamos calificar de pobreza. Por cabeceras comarcales el porcentaje de "impotentes" fue más reducido en San Vicente de la Sonsierra (14,62\%), que en Laguardia (22,33\%), Viana (22,33\%) y Labraza $(21,42 \%)$.

Por otra parte los cabezas de familia no fueron hombres en todos los casos. Aunque no se aprecia en los cuatro términos jurisdiccionales un porcentaje elevado de fuegos de población femeninos. Un tanto por ciento de casas dirigidas por mujeres superior al $15 \%$ ha sido valorado por algunos demógrafos como una consecuencia del efecto negativo provocado por una grave epidemia. En estas circunstancias se ha propuesto la conveniencia de contabilizar un menor número de personas por fuego habitado ${ }^{17}$. En 1427 el número de fuegos feme-

16. El número de casas abandonadas se cifra en 1.124, pero si estimamos las casas que estuvieron levantadas en las aldeas despobladas dicha cuantía ascendería hasta 1.259.

17. BILBAO, L. Ma., y FERNÁNDEZ DE PINEDO, E. "En torno al problema del poblamiento y la población del País Vasco en la Edad media". Homenaje a Julio Caro Baroja. Madrid, 1978, pp. 131-160. 
ninos no llega al 9\% del conjunto de los censados en la Sonsierra de Navarra. Viana y sus aldeas son las localidades donde el porcentaje es más elevado, con el $14,56 \%$ de las casas de la jurisdicción. Igualmente el porcentaje de fuegos "polinucleares", es decir, de casas donde residen padres con hijos casados o dos unidades familiares diferenciadas es bastante bajo. No llega al $3 \%$ de los hogares censados, lo que denota la importancia en esta comarca de células de encuadramiento de carácter unifamiliar.

\section{La presión fiscal ejercida en 1427 sobre los pobladores de San Vicente, Laguardia, Labraza y Viana}

La administración del reino de Navarra designó a varias personas para elaborar el censo fiscal de 1427. Los "comisionados" para la colecta fueron los encargados de gestionar las demandas de "ayudas" y "cuarteles". Entre sus fines principales sobresalen los de recorrer las villas y aldeas que debían pagar dichos tributos y recaudar las cantidades globales estipuladas. Se ocuparon asimismo de poner por escrito la situación socioeconómica de cada una de las localidades por las que transitaron, de recoger las quejas de los pobladores, de anotar las gracias y privilegios que presuntamente habían recibido de los reyes navarros y de señalar el montante de los tributos cotizados en los años precedentes. Sin embargo, la distribución de la recaudación entre los vecinos con casa no fue función de los comisarios. Los concejos tenían sus propias comisiones que distribuyeron la cantidad a sufragar a partir del patrimonio y de los bienes de los contribuyentes. Este sistema impositivo no estuvo exento de posibles omisiones y fraudes.

No se tiene noticia de las bases patrimoniales que sustentaron el reparto del impuesto entre el número de hogares de las jurisdicciones de Laguardia, San Vicente, Labraza y Viana. Es muy probable que los baremos fiscales aplicados no fueran los mismos en los cuatro términos jurisdiccionales señalados. Pese a todo, el examen del censo fiscal permite establecer una serie de comparaciones entre las cuatro villas y sus aldeas. De hecho, es posible conocer la gradación impositiva a que estuvieron sometidos los vecinos y moradores de la Sonsierra. Desde luego, los pobladores de Laguardia y sus aldeas fueron quienes más tributaron en conjunto, con 153.601 dineros. Cifra muy superior a las de Viana y sus aldeas, con 90.404 dineros, San Vicente y sus aldeas, con 41.520 dineros y Labraza con sus aldeas, que apenas tributaron 19.512 dineros. Estas cantidades globales deben ser contempladas en relación con el número de hogares de cada una de estas comarcas. De la división del total de los dineros de la recaudación con el número de "fuegos de población" de cada comarca resulta la presión fiscal media ejercida en Laguardia, San Vicente, Labraza y Viana. 
Fiscalidad en la Sonsierra de Navarra, según el censo de 1427

\begin{tabular}{|l|c|c|}
\hline & $\begin{array}{c}\text { MONTANTE TOTAL DEL TRIBUTO } \\
\text { (EN DINEROS) }\end{array}$ & $\begin{array}{c}\text { PRESIÓN MEDIA FISCAL EN CADA } \\
\text { JURISDICCIÓN (EN DINEROS) }\end{array}$ \\
\hline Laguardia y sus aldeas & 153.601 & 291,46 \\
\hline San Vicente y sus aldeas & 41.520 & 164,11 \\
\hline Labraza y sus aldeas & 19.512 & 694,08 \\
\hline Viana y sus aldeas & 90.404 & 438,85 \\
\hline
\end{tabular}

Realizada dicha operación matemática es evidente, de forma aparentemente paradójica, que los contribuyentes más penalizados fiscalmente fueron los de Labraza y sus dos aldeas de Barriobusto y Villavieja. Estos soportaron una media de 694,08 maravedís por vecino, frente a los 438,85 de Viana con sus aldeas, los 291,46 de Laguardia con sus aldeas y los 164,11 maravedís de San Vicente con sus aldeas, los vecinos menos gravados fiscalmente. El conjunto de los vecinos de la Sonsierra contribuyeron en total con 305.037 dineros, siendo la media general del impuesto 300,82 dineros. Por debajo de dicha media tributaron las comunidades de Laguardia y San Vicente, circunstancia que está relacionada con las mayores exenciones y gracias fiscales otorgadas por la monarquía navarra. Probablemente también estos resultados deban relacionarse con el desequilibrio demográfico operado en cada uno de estos centros político-administrativos, con las guerras y con las negociaciones planteadas por los concejos urbanos para reducir el pago de impuestos.

Cabría preguntarse si la recesión demográfica y los efectos de las guerras con Castilla, acaecidos entre principios del siglo XIV y finales del primer tercio del siglo XV, fueron superiores en Laguardia y San Vicente que en Viana y Labraza, a tenor de la menor presión fiscal soportada por las dos primeras. Es probable, pero no es fácil resolver esta cuestión de una manera definitiva. Igualmente es útil comparar la carga fiscal de la Sonsierra con otras zonas del Reino. A este respecto los burgos de Estella estuvieron sometidos a una presión fiscal media de 266,42, cantidad mucho más baja que la existente en la Sonsierra del Reino de Navarra. La tasa fiscal media de los burgos de Pamplona fue todavía mucho más pequeña, 163,63 dineros ${ }^{18}$. Que San Vicente y Laguardia estuvieran en una zona fronteriza, por tanto, no fue motivo suficiente para que se redujera la presión fiscal a sus pobladores. Incluso Viana y Labraza, zonas muy próximas a la frontera castellana, soportaron cargas fiscales bastante elevadas. Por el contrario, en Estella y Pamplona, disfrutaron de unas condiciones impositivas mucho más favorables.

18. Véase GARCÍA FERNÁNDEZ, E. "Fiscalidad y sociedad en la Pamplona medieval (1427$1435)^{\prime \prime}$...etc., y del mismo autor "Fiscalidad y niveles de renta de la población de Estella a comienzos del siglo $\mathrm{XV}{ }^{\prime \prime}$... etc. 
Presión fiscal media, eliminados los hogares que no cotizaron

\begin{tabular}{|l|c|c|}
\hline & $\begin{array}{c}\text { NÚMERO DE CONTRIBUYENTES } \\
\text { REALES }\end{array}$ & $\begin{array}{c}\text { PRESIÓN MEDIA FISCAL EN CADA } \\
\text { JURISDICCIÓN (EN DINEROS) }\end{array}$ \\
\hline Laguardia y sus aldeas & 406 & 378,32 \\
\hline San Vicente y sus aldeas & 216 & 192,22 \\
\hline Labraza y sus aldeas & 22 & 886,90 \\
\hline Viana y sus aldeas & 160 & 565,02 \\
\hline
\end{tabular}

Dejando al margen el número de hogares "impotentes", es decir, si restamos aquellas personas con casa que no tributaron finalmente en cada jurisdicción, los contribuyentes reales disminuyen en las cuatro comarcas estudiadas y la presión fiscal media se ve incrementada en todos los casos. Esta operación depara una mayor aproximación a la nueva media fiscal (379,39 dineros) de los pobladores de Laguardia y sus aldeas, un alejamiento considerable de los de San Vicente y una superación notable de la tasa media de los vecinos de Labraza y Viana.

Tributación de los clérigos de la Sonsierra de Navarra (año 1427)

\begin{tabular}{|l|c|c|c|}
\hline & Número de clérigos & Dineros pagados & $\begin{array}{c}\text { Presión media fiscal } \\
\text { (en dineros) }\end{array}$ \\
\hline Laguardia y sus aldeas & 21 & 8.205 & 390,71 \\
\hline San Vicente y sus aldeas & 14 & 3.360 & 240 \\
\hline Labraza y sus aldeas & 3 & 4.170 & 1.390 \\
\hline Viana y sus aldeas & 21 & 9.729 & 463,28 \\
\hline TOTAL & 59 & 25.464 & 431,59 \\
\hline
\end{tabular}

No es de menor relevancia detenerse, siquiera brevemente, en el estudio de aquellos sectores sociales sobre los que recayó fundamentalmente el impuesto. Desde esta perspectiva de análisis los 59 clérigos referenciados pagaron una media de 431,59 dineros, si bien variaron bastante de una a otra jurisdicción. En Labraza 1.390 dineros, en Viana 463,28 dineros, en Laguardia 390,71 dineros y San Vicente 240 dineros. El conjunto de los laicos citados, por el contrario, tributaron una media de 292,74, cuantía substancialmente menor a la de los beneficiados eclesiásticos. Este dato nos da una primera imagen del nivel de vida de los clérigos locales. Estos tributaban unos 138,85 maravedís más que la media general de todos los pobladores de la Sonsierra. Lógicamente los eclesiásticos, de manera individualizada, debieron contar de forma porcentual con más bienes patrimoniales que el resto de la gente. Representando el 5,81\% de los "fuegos de 
población" recogidos en el censo de 1427, contribuyeron, no obstante, con el $8,34 \%$, es decir, 2,53 puntos por encima del porcentaje que significaban.

Entre los "laicos tributarios" sobresalen los notarios, cuya media general del impuesto supuso 470,76 dineros, 39 puntos por encima de los clérigos. Quienes más pagaron fueron los cuatros notarios de Viana, 3.246 dineros, es decir, 856,5 dineros de media. A continuación los cuatro notarios de Laguardia contribuyeron con 1.554 dineros, es decir, 388,5 dineros de media. Los cinco notarios de San Vicente fueron tasados con 1.320 dineros, es decir, 264 dineros de media. Estamos en presencia de una serie de individuos cultos y cualificados, que alcanzaron unos niveles de fortuna altos en la Sonsierra del reino de Navarra. Las diferencias fiscales entre los notarios de Laguardia, San Vicente y Viana están relacionadas con la divergente distribución del tributo de los "cuarteles" existente entre los pobladores con casa en cada una de estas circunscripciones político-administrativas.

De igual modo, quienes desempeñaron este año el oficio de alcalde fueron repartidos en cantidades bastante más elevadas que la media general de cada una de las villas y aldeas. No es infrecuente, además, que ellos mismos formen parte de los comisionados encargados de llevar a efecto la distribución de las libras, sueldos y dineros que habían de pagar los vecinos sobre quienes tenían poderes de carácter jurisdiccional, como jueces designados por el rey de Navarra. El alcalde de Laguardia fue tasado en 396 dineros, el de Viana en 1.451 dineros, el de San Vicente en 240 dineros y el de Labraza en 1.092 dineros. La media general del tributo de los cuatro alcaldes alcanza los 794,75 dineros. De este dato se infiere que riqueza y poder iban bastante parejos, aunque de ello no se deriva necesariamente que en todas las zonas fueran siempre los más ricos quienes gobernaron.

Tributación de los fuegos de población femenina en la Sonsierra de Navarra, según el censo fiscal de 1427

\begin{tabular}{|l|c|c|c|}
\hline & Número de mujeres & Dineros pagados & $\begin{array}{c}\text { Presión media fiscal } \\
\text { (en dineros) }\end{array}$ \\
\hline Laguardia y sus aldeas & 37 & 7.534 & 203,62 \\
\hline San Vicente y sus aldeas & 22 & 4.260 & 193,63 \\
\hline Labraza y sus aldeas & 1 & 180 & 180 \\
\hline Viana y sus aldeas & 30 & 14.295 & 476,5 \\
\hline TOTAL & 90 & 26.269 & 291,87 \\
\hline
\end{tabular}

Los hogares constituidos por mujeres significaron el $8,87 \%$ del total de los recogidos en el censo de 1427 . Su cotización supuso unos porcentajes similares, el 8,61\%. La presión fiscal media de dichos hogares fue de 291,87 dineros. 
Esta cifra no está lejos de la media general $(300,82)$, tan sólo 8,95 puntos por debajo ${ }^{19}$. No obstante, en dicho cómputo se han incluido los "fuegos polinucleares" con presencia de mujeres. Si restamos los "fuegos polinucleares" la situación cambia de forma notable. Contaríamos con 7 "fuegos de población" femenina menos, 83, que contribuyen con 23.789 dineros. Las mujeres representan de este modo el $8,18 \%$, cotizan con el $7,79 \%$ y pagan una media de 286,61 , es decir, 14,21 puntos menos que la media general de toda la Sonsierra. La suma en dineros de los siete "fuegos polinucleares", a cuyo frente se encontraba una mujer asciende a 2.480 dineros. La media fiscal tributaria alcanzaba los 354,28 dineros.

¿Es posible saber sobre qué grupos de fortuna recayó mayoritariamente el impuesto recaudado en 1427 ?. El acercamiento a esta temática requiere la distribución de la población por niveles socioeconómicos. Sabemos que era un impuesto bastante progresivo. Los más ricos pagaban más y quienes carecían de bienes estaban exentos.

En función del volumen impositivo exigido a cada uno de los contribuyentes he establecido 7 niveles socioeconómicos. Estos son los que tributan entre las siguientes cantidades: de 0 a 75 dineros, de 76 a 150 dineros, de 151 a 225 dineros, de 226 a 300 dineros, de 301 a 375 dineros, de 376 a 450 dineros y aquellos que pagan más de 450 dineros. Ésta es la base a partir de la cual he tratado de concretar el nivel de fortuna de los hogares de la Sonsierra de Navarra. No hay que pasar por alto, sin embargo, el hecho de que la distribución de los dineros a recaudar seguía a su vez los criterios establecidos respectivamente en los concejos de San Vicente, Laguardia, Labraza y Viana. Estas poblaciones dispusieron con toda seguridad de libros de estimas de los bienes patrimoniales de sus habitantes. En ellos debía descansar de manera fundamental la posterior estimación fiscal a que estaba sujeto cada hogar. Los gráficos realizados al efecto e incorporados en el apéndice son un buen ejemplo del porcentaje de población que representa cada nivel fiscal considerado y de la presión fiscal a que estuvieron sometidos estos pobladores. Es palpable en todos los casos que el impuesto fue soportado por los más ricos, si bien no con la misma intensidad en las cuatro circunscripciones político-administrativas.

Ya a finales del siglo XIV en algunas localidades del reino de Navarra se distinguía entre hogares "mayores", "medianos" y "menores". A mi entender en 1427 se podrían agrupar aún más los 7 niveles que he establecido, hasta reducirlos a cuatro. Un primer grupo, que incluye a las personas más acomodadas, comprendería a quienes estaban tasados con más de 450 dineros, un segundo grupo englobaría a quienes se hallaban entre los 226 y 450 dineros -los grupos medianos-, un tercero a aquellos que estaban entre 76 y 225 dineros -los

19. En Viana y en San Vicente la carga fiscal de las mujeres fue superior a la del conjunto de los "fuegos de población". 
menores- y un cuarto, los que apenas tienen bienes o son muy pobres. La mayoría de los componentes de este último grupo fue eximido del pago del tributo por su condición social más paupérrima.

Los resultados reflejados en los gráficos incorporados en el apéndice demuestran que en el conjunto de la Sonsierra del Reino de Navarra quienes cotizaron con más de 450 dineros supusieron el 25,24\% de los hogares. Contribuyeron con el $62,89 \%$ del impuesto. Los grupos "medios" alcanzaban el $26,91 \%$ y pagaron el $26,25 \%$. Los estimados como grupos "menores", representaban el 21,37\% y tan sólo tributaban con el 9,8\%. Los propiamente "pobres", el 26,42\%, apenas fueron tasados con el 1,04\% del tributo. Estos datos ya nos ofrecen una primera muestra del reparto de la presión fiscal en la Sonsierra, pero los matices aparecen al analizar cada una de las circunscripciones. La villa de Laguardia con sus aldeas se mueve básicamente en los parámetros numéricos señalados previamente: los "mayores" son el 25,99\% y pagan el $61,63 \%$, los "medianos" son el $26,36 \%$ y pagan el $27,45 \%$, los "menores" son el $19,72 \%$ y tributan el $10,08 \%$, mientras que los propiamente "pobres" son el $27,89 \%$ y cotizan el $0,81 \%$.

Sin embargo los contrastes se producen sobre todo entre Viana y San Vicente. En San Vicente los "mayores" suponen el 11,46\% y pagan el 33,52\%, los "medianos" el 23,32\% y cotizan el 34,1\%, los "menores" son el 37,94\% y pagan el $27,74 \%$, mientras que los "pobres" son el $27,70 \%$ y pagan el 4,62\%. En Viana por el contrario el grupo de los sectores sociales más acomodados es mayor y cotiza más, el 36,4\% pagando el 73\%, los "medianos" siendo el $33,96 \%$ sufragan el $23,49 \%$, los "menores" representan el 7,27\% y pagan el $2,77 \%$, mientras que el $22,33 \%$ de "pobres" no fue tasado en cantidad alguna. Está meridianamente claro que en Viana la mayoría de la población fue distribuida entre los "mayores" y "los medianos". Ambos grupos sostienen casi todo el tributo, el 96,49\%. En San Vicente "mayores" y "medianos" cotizaron el $67,62 \%$, porcentaje que en Laguardia se incrementó hasta el 89,08\%.

Labraza todavía evidencia con más intensidad las diferencias entre los vecinos tipificados como ricos y los demás. Pues los primeros, el 53\%, pagan el $88,46 \%$ del tributo, los pobres, el $21,42 \%$, no fueron tasados en cantidad alguna, los "medianos", el 17,85\%, significaron el 9,52\% y los "menores", el $7,14 \%$, debían sufragar el 1,99\%. En esta última comarca, sin embargo, Ilaman la atención dos cosas: en primer lugar el escaso número de "fuegos de población" (28 en total) y en segundo lugar que la mayoría de la población -15 hogares- fue incluida en el nivel fiscal superior.

\section{Conclusiones}

Las valoraciones históricas expresadas en el artículo se sustentan en gran medida en los datos recogidos en cuadros y gráficos incorporados en el texto y en el apéndice de gráficos, cuadros y documental. Como colofón del artículo 
voy a señalar algunas de las hipótesis de trabajo sostenidas y desarrolladas en el mismo.

La evolución demográfica de Laguardia, San Vicente, Labraza y Viana con sus respectivas aldeas sufrió alteraciones diferenciadas en el curso del siglo XIV y de los primeros años del siglo XV. Basta fijarse a este respecto en el número de despoblados y en el de las supuestas casas abandonadas por sus pobladores. Ambos datos son recogidos en el censo de la Merindad de Estella del año 1427, resultado de la "ayuda" solicitada por los reyes Juan II y doña Blanca de Navarra. Es seguro que las carestías, la peste negra, las epidemias posteriores y las guerras entre Castilla y Navarra hicieron mella entre los moradores de la Sonsierra del Reino de Navarra. No tuvieron, sin embargo, los mismos efectos fiscales en el conjunto de la Sonsierra.

La menor presión fiscal existente en San Vicente y Laguardia, en comparación con las poblaciones de Viana y Labraza, parece ser otro síntoma más de las complejas y variadas vicisitudes por las que atravesaron los habitantes de estos espacios navarros ribereños del Ebro, frontera fluvial entre los reinos de Castilla y de Navarra por aquellas fechas. Asimismo se aprecia la existencia de un reparto desigual del tributo entre los pobladores de la Sonsierra y de una presión fiscal que no es homogénea, ni uniforme. La distribución de la "ayuda" solicitada tuvo especificidades en cada una de las cuatro jurisdicciones territoriales.

Pero los más ricos y acomodados soportaron mayoritariamente el impuesto de 1427. Los alcaldes, los clérigos y los notarios fueron quienes contribuyeron en término medio con sumas económicas superiores al resto de los sectores sociales. Quienes más patrimonio disponían cotizaban más. ¿Ahora bien, de acuerdo con su riqueza, podían haber contribuido aún más comparativamente con los menos favorecidos?. Es probable. De hecho el reparto del "cuartel", principalmente en Laguardia y Viana, fue soportado mayoritariamente por quienes tributaron más de 451 dineros. Por el contrario en San Vicente de la Sonsierra la mayor parte del impuesto fue costeado por quienes cotizaron entre 376 y 450 dineros. 


\section{Apéndice de gráficos}

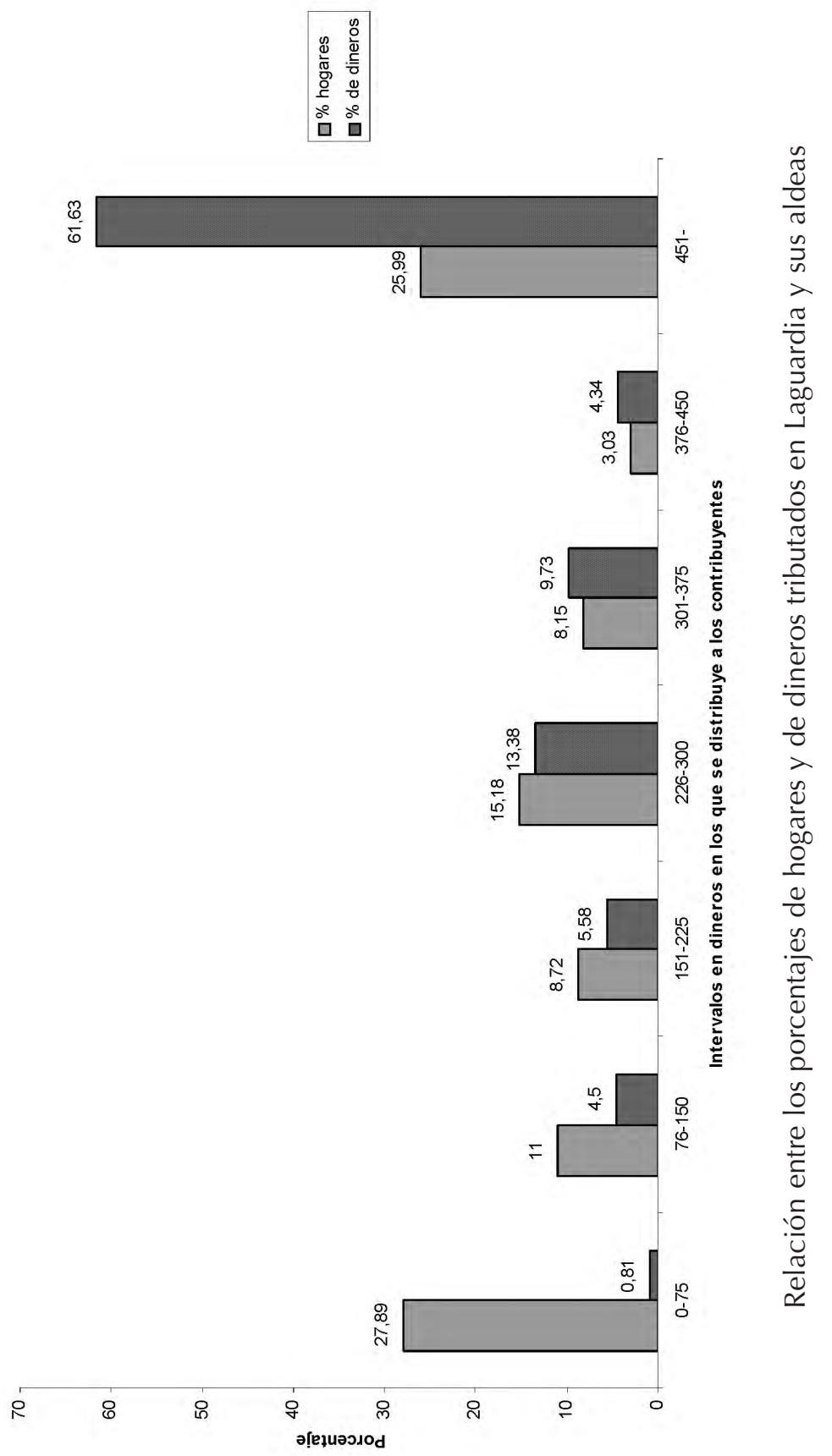


FISCALIDAD, DEMOGRAFÍA Y SOCIEDAD EN LA SONSIERRA EN TORNO A 1427...

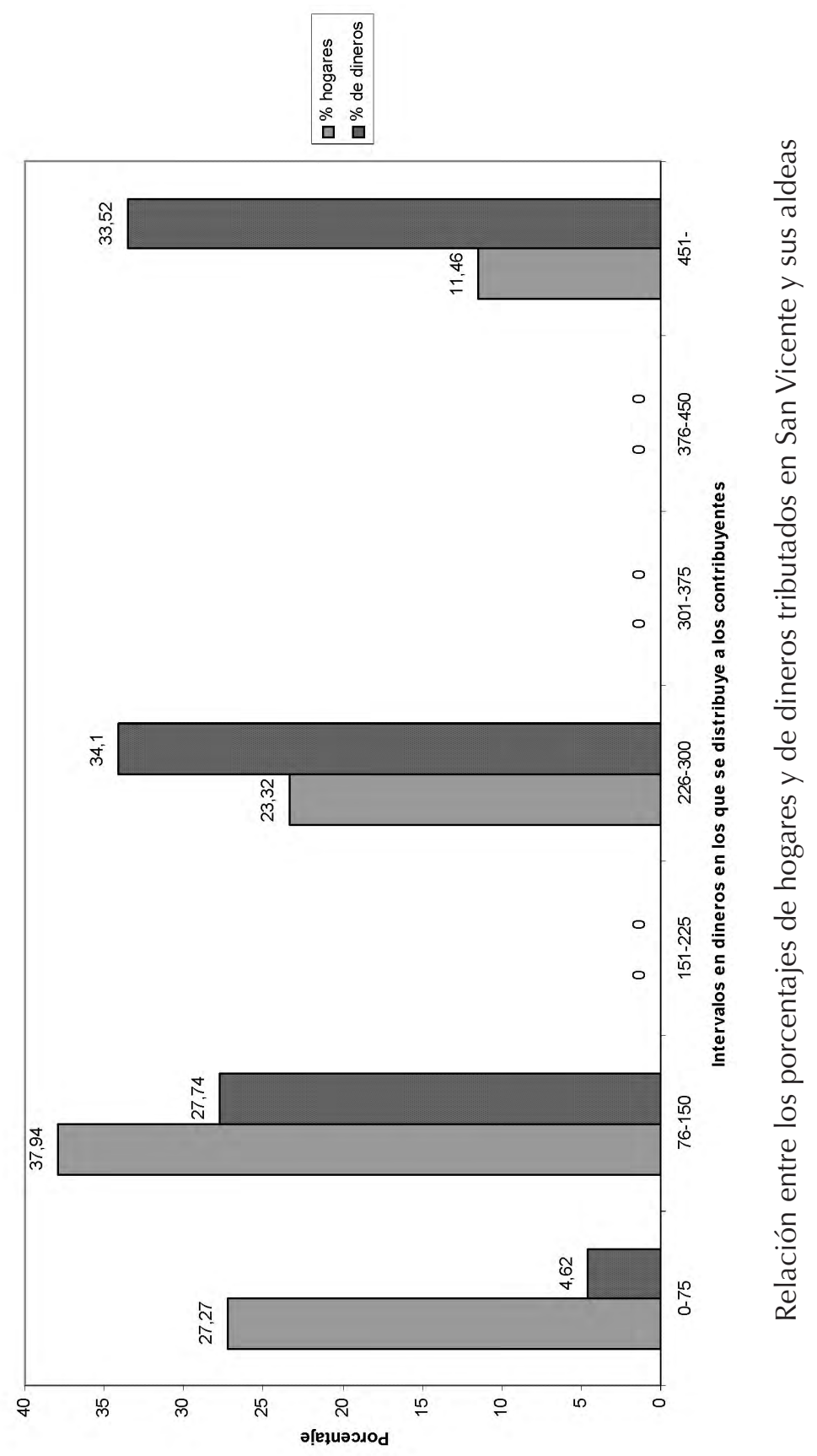


ERNESTO GARCÍA FERNÁNDEZ

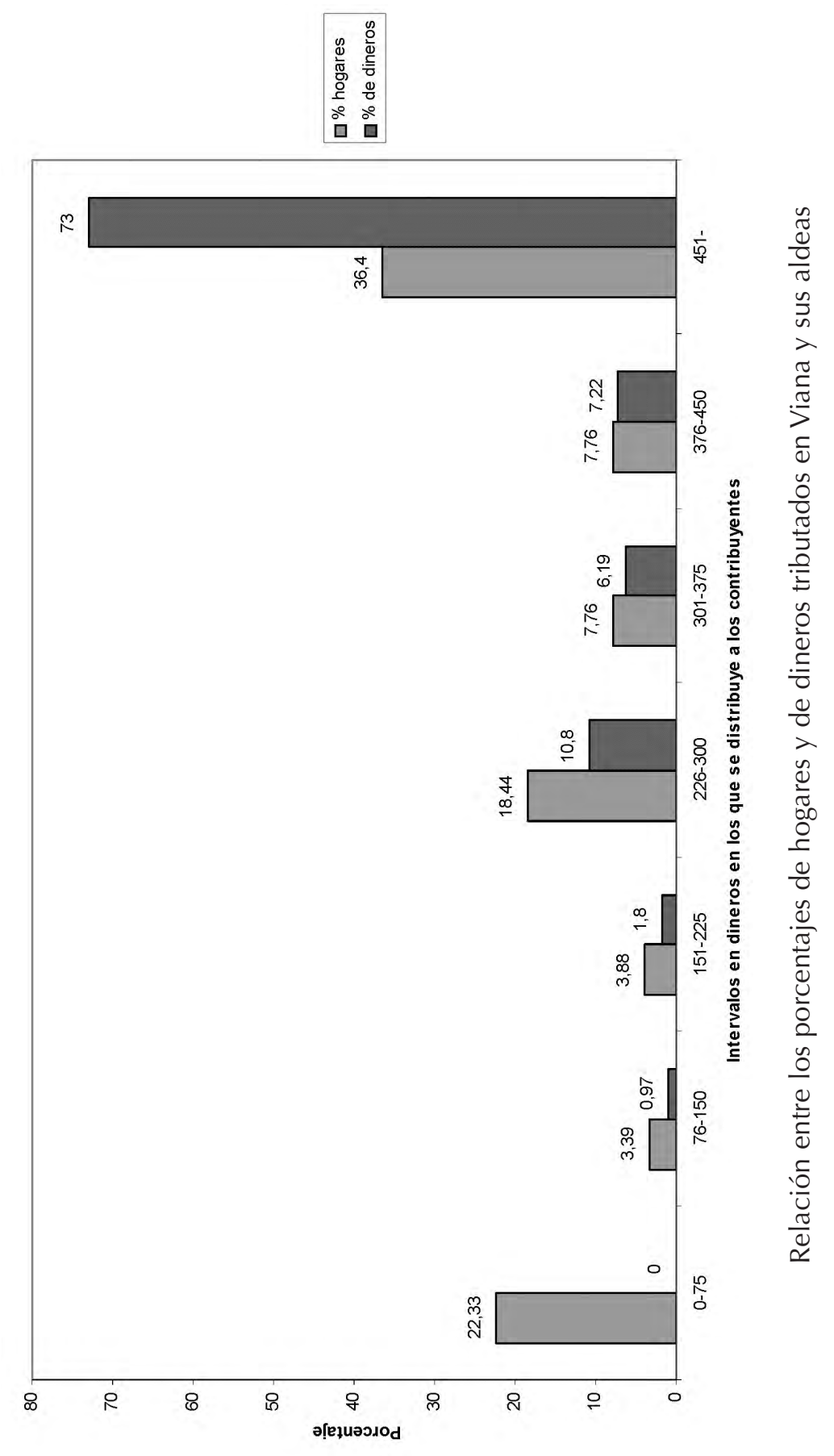


FISCALIDAD, DEMOGRAFÍA Y SOCIEDAD EN LA SONSIERRA EN TORNO A 1427...

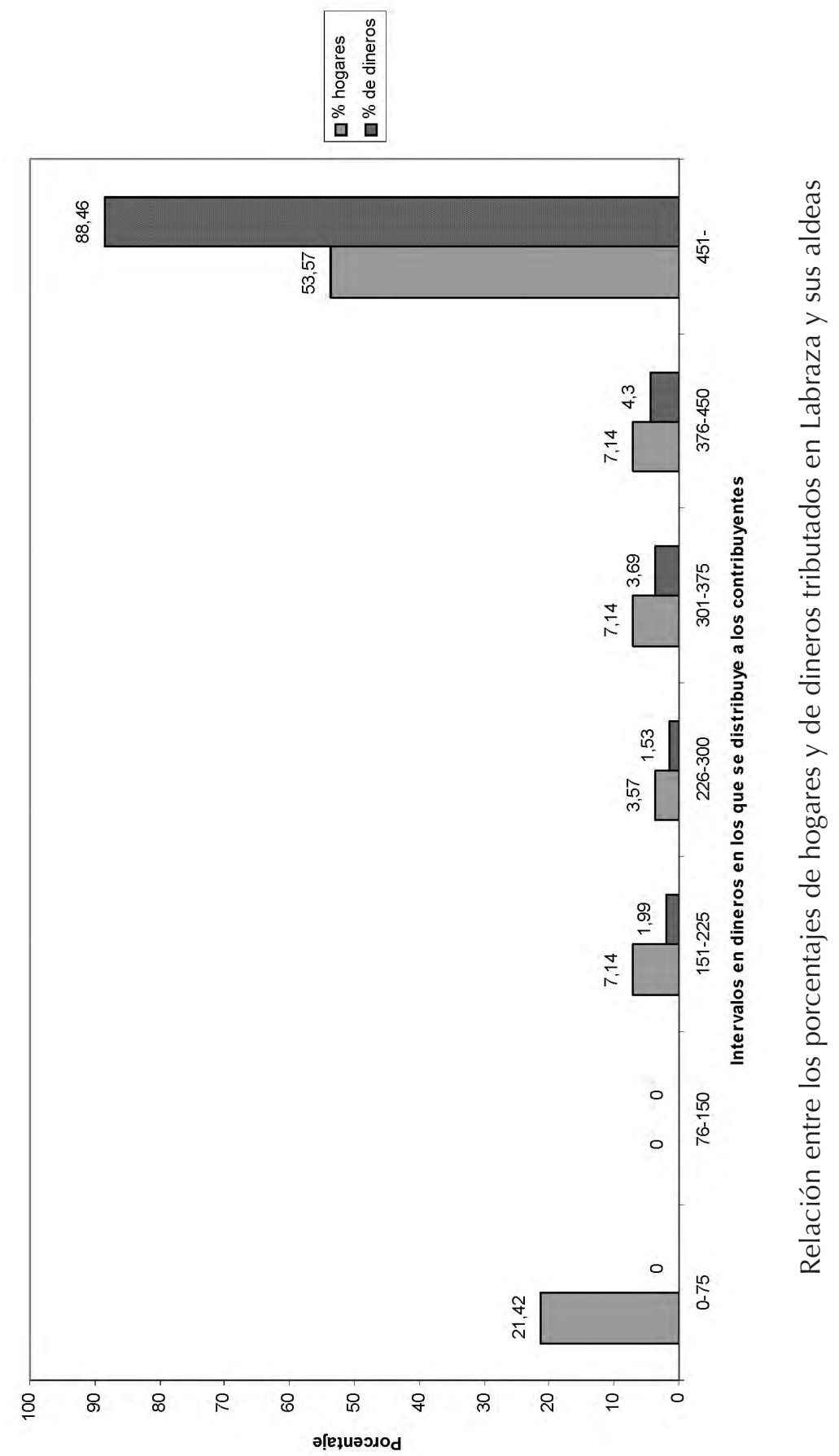


ERNESTO GARCÍA FERNÁNDEZ

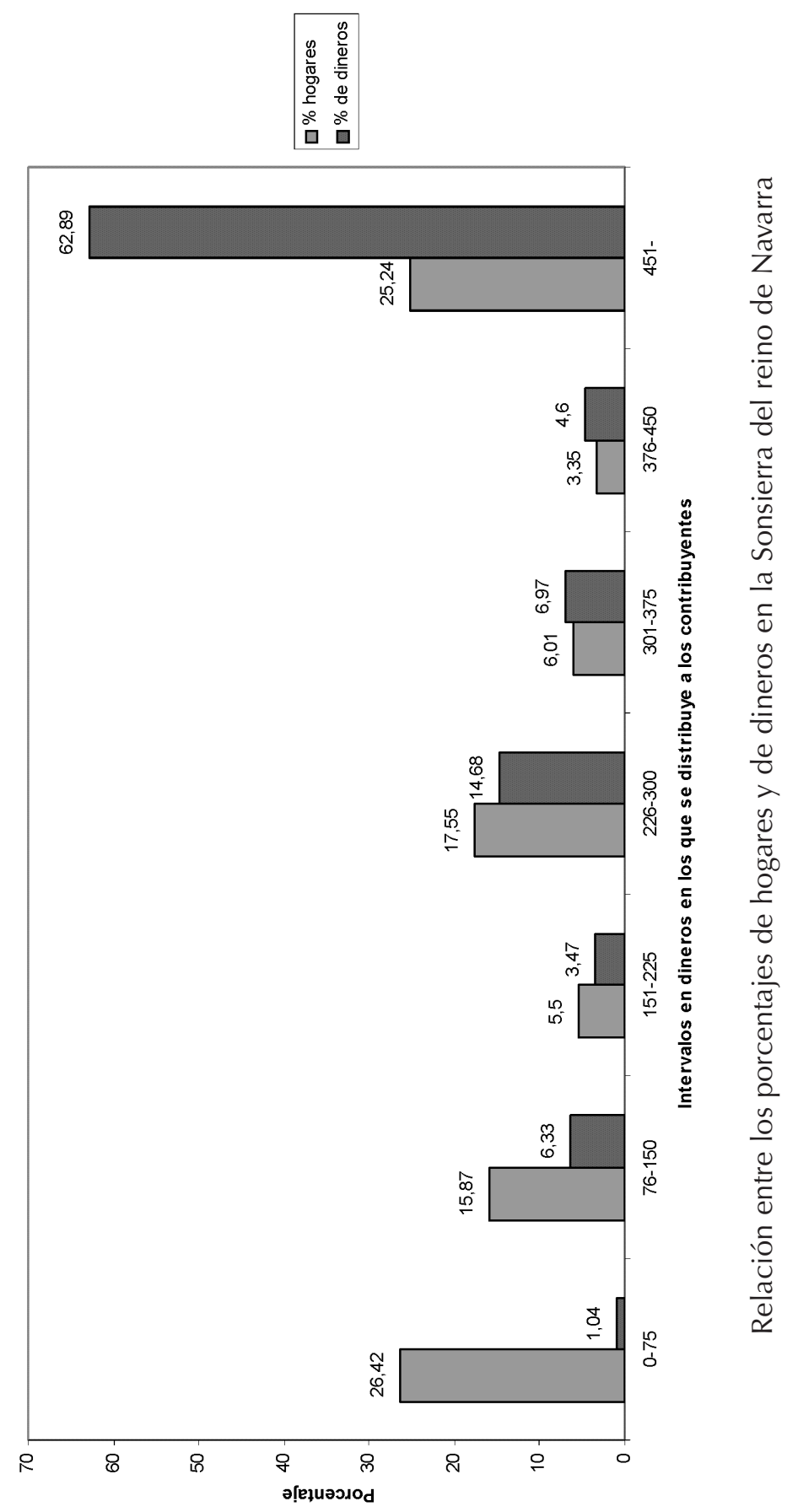




\section{Apéndice de cuadros}

CUADRO No 1 . Datos de la villa y aldeas de laguardia, según el censo fiscal de 1427

\begin{tabular}{|l|r|r|c|c|c|c|c|c|}
\hline & Casas & Dineros & $\begin{array}{c}\text { Media de } \\
\text { la presión } \\
\text { fiscal por } \\
\text { casa }\end{array}$ & $\begin{array}{l}\text { Impotentes } \\
\text { Laguardia }\end{array}$ & $\begin{array}{c}\text { Casas } \\
\text { abandonadas }\end{array}$ & Mujeres & Polinucleares & Clérigos \\
\hline Elvillar & 64 & 49.031 & 298,96 & 52 & 280 & 5 & 1 & 7 \\
\hline Elciego & 20 & 5.430 & 271,5 & 2 & 14 & 2 & - & 1 \\
\hline Samaniego & 32 & 7.908 & 247,12 & 4 & 22 & 6 & - & 2 \\
\hline Baños & 12 & 1.206 & & 4 & 8 & - & - & - \\
\hline Leza & 18 & 5.964 & 100,5 & 3 & 7 & 2 & 4 & 1 \\
\hline Páganos & 10 & 2.216 & 331,33 & 1 & 7 & - & 1 & - \\
\hline Cripán & 39 & 11.928 & 305,84 & 12 & 27 & 1 & - & 1 \\
\hline Viñaspre & 8 & 1.482 & 185,25 & 2 & 31 & 1 & 2 & - \\
\hline Yécora & 26 & 10.956 & 421,38 & 8 & 18 & 1 & 2 & 3 \\
\hline Lanciego & 29 & 11.364 & 391,86 & 9 & 16 & 2 & - & 1 \\
\hline Navaridas & 17 & 4.140 & 243,52 & 2 & 5 & 2 & 1 & 1 \\
\hline Pazuengos & 2 & 228 & 114 & - & - & - & - & - \\
\hline Oyón & 25 & 8.574 & 342,96 & 5 & 20 & - & 2 & 1 \\
\hline La puebla & 9 & 3.522 & 391,33 & - & - & - & - & - \\
\hline Moreda & 14 & 2.796 & 199,71 & 3 & 29 & 2 & 1 & 2 \\
\hline $\begin{array}{l}\text { Villascuerna } \\
\text { (Villabuena) }\end{array}$ & 16 & 4.740 & 296,25 & 3 & 8 & - & - & 1 \\
\hline Berberana & 12 & 2.868 & 239 & 3 & 22 & 2 & 2 & - \\
\hline Quintana & 10 & 1.638 & 163,8 & 3 & 10 & - & - & - \\
\hline $\begin{array}{l}\text { Total villa y } \\
\text { aldeas }\end{array}$ & 527 & 153.601 & 291,46 & 121 & 569 & 37 & 16 & 21 \\
\hline
\end{tabular}


CuAdro No 2. Datos de la villa y aldeas de San Vicente de la Sonsierra, según el censo fiscal de 1427

\begin{tabular}{|l|c|c|c|c|c|c|c|c|}
\hline & Casas & Dineros & $\begin{array}{c}\text { Media de } \\
\text { la presión } \\
\text { fiscal por } \\
\text { casa }\end{array}$ & Impotentes & $\begin{array}{c}\text { Casas } \\
\text { abandonadas }\end{array}$ & Mujeres & Polinucleares & Clérigos \\
\hline $\begin{array}{l}\text { Villa de } \\
\text { San Vicente }\end{array}$ & 139 & 22.680 & 163,16 & 20 & 240 & 14 & 7 & 10 \\
\hline Ávalos & 74 & 11.100 & 150 & 14 & 50 & 6 & 3 & 4 \\
\hline Peciña & 18 & 3.480 & 193,33 & 3 & 5 & - & - & - \\
\hline Orzales & 12 & 2.580 & 215 & - & 4 & 1 & - & - \\
\hline Ribas & 10 & 1.680 & 168 & - & 2 & 1 & - & - \\
\hline $\begin{array}{l}\text { Total villa y } \\
\text { aldeas }\end{array}$ & 253 & 41.520 & 164,11 & 37 & 301 & 22 & 10 & 14 \\
\hline
\end{tabular}

CuAdro No ${ }^{\circ}$. Datos de la villa y aldeas de Viana, según el censo fiscal de 1427

\begin{tabular}{|l|r|r|c|c|c|c|c|c|}
\hline & Casas & Dineros & $\begin{array}{c}\text { Media de } \\
\text { la presión } \\
\text { fiscal por } \\
\text { casa }\end{array}$ & Impotentes & $\begin{array}{c}\text { Casas } \\
\text { abandonadas }\end{array}$ & Mujeres & Polinucleares & Clérigos \\
\hline Villa de Viana & 178 & 78.314 & 439,96 & - & 171 & 26 & - & 21 \\
\hline Aras & 5 & 1.266 & 253,2 & - & 30 & 3 & - & - \\
\hline Bargota & 16 & 8.994 & 562,12 & - & 16 & 1 & - & - \\
\hline Cuevas & 3 & 870 & 290 & - & 10 & - & - & - \\
\hline Lazagurría & 4 & 960 & 240 & - & - & - & - & - \\
\hline $\begin{array}{l}\text { Total villa y } \\
\text { aldeas }\end{array}$ & 206 & 90.404 & 438,85 & 46 & 227 & 30 & - & 21 \\
\hline
\end{tabular}


CuAdro N ${ }^{0}$ 4. Datos de la villa y aldeas de Labraza, según el censo fiscal de 1427

\begin{tabular}{|l|r|r|c|c|c|c|c|c|}
\hline & Casas & Dineros & $\begin{array}{c}\text { Media de } \\
\text { la presión } \\
\text { fiscal por } \\
\text { casa }\end{array}$ & Impotentes & $\begin{array}{c}\text { Casas } \\
\text { abandonadas }\end{array}$ & Mujeres & Polinucleares & Clérigos \\
\hline $\begin{array}{l}\text { Villa de } \\
\text { Labraza }\end{array}$ & 25 & 17.352 & 694,08 & 6 & & 1 & 2 & 3 \\
\hline Barriobusto & 2 & 720 & 360 & - & & - & - & - \\
\hline Villavieja & 1 & 1.440 & 1.440 & - & & - & - & - \\
\hline $\begin{array}{l}\text { Total villa y } \\
\text { aldeas }\end{array}$ & 28 & 19.512 & 696,85 & 6 & 27 & 1 & 2 & 3 \\
\hline
\end{tabular}

CuADRO $N^{0}$ 5. Datos generales sobre las comunidades de Villa y aldeas de San Vicente, Laguardia, Labraza y Viana según el censo fiscal de 1427

\begin{tabular}{|c|c|c|c|c|c|c|c|c|}
\hline & Casas & Dineros & $\begin{array}{c}\text { Media de } \\
\text { la presión } \\
\text { fiscal por } \\
\text { casa }\end{array}$ & Impotentes & $\begin{array}{c}\text { Casas } \\
\text { abandonadas }\end{array}$ & Mujeres & Polinucleares & Clérigos \\
\hline & 1.014 & 305.037 & 300,82 & 210 & 1.124 & 90 & 28 & 59 \\
\hline
\end{tabular}

\section{Apéndice documental}

Fuegos de la villa y aldeas de San Vicente de la Sonsierra de 1427.

Archivo General de Navarra. Libro de Fuegos de la Merindad de Estella del año 1427, folios 163-170, S/S.

La villa de Sant Bicent con sus aldeas que son Davallos Pizinna Orçales et Riba.

Primo nos los dichos comisarios reçebimos jura sobre la cruz e sanctos evangelios toquandolos manualmente destos vecinos de la billa de Sant Bicent que se siguen.

Primo de don Johan cura de la dicha billa.

Item de Martín Sanchiz de Doroynno alcalde de la dicha billa.

Item de Martín Sanchiz de los tenderos notario.

Item de Sancho Lopiz fijo de Diago Lopiz regidores de la dicha billa. 
Et sobre la dicha jura interrogados quantos moradores casa mantenientes ay de clerigos e legos ay en la dicha billa de Sant Biçent dixieron que ay los que se siguen

Primo el dicho don Johan cura

$\mathrm{XL} \mathrm{s}$.

Item don Johan Sanchiz Lorent arcipreste de Lagoardia

$\mathrm{XLS}$.

Item don Roy Martiniz

$X L s$.

Item don Ferrant Periz capeillan

$X X s$.

Item el dicho don Roy Martiniz e Martin Sanchiz por los bienes del panno

XX s.

Item don Ferrando Diaz cura

XXs.

Item el dicho don Martín Sanchiz por sus bienes

$X X s$.

Item don Martín Sanchiz fijo de Ferrant Sanchiz

$X \mathrm{~s}$.

Item don Martín Ferrandiz fijo de Johan Ferrandiz

$X s$.

Item don Pedro Sanchiz de Garixuri

$\mathrm{X}$ s.

\section{Legos}

Primo el dicho Martín Sanchiz fijo de Johan Sanchiz de Doroynno alcalde $\quad$ XX s.

Item el dicho Sancho Lopiz fijo de Diago Lopiz XL s.

Item Martín Sanchiz notario XX s.

Item Johan Alfonso XL s.

Item Gonçalo fijo de Sancho Martiniz $\quad$ XL s.

Item Sancho fijo de Ferrant Lopiz XL s.

Item Johan Martiniz fijo de Gonçalo Martiniz $\quad$ XL s.

Item Gonçalvo su sobrino $\quad X L$ s.

Item Johan Sanchiz de Doroyno XL s.

Item la muger de Johan Sanchiz de Garixuri $\quad$ XL s.

Item Johan Martiniz pelegrin $\quad$ XL s.

Item Martin fijo de Martin Ferrandiz $\quad$ XL s.

Item Johan Ruyz con su madre $\quad X L$ s.

Item Johan Ferrandiz de Garixuri XL s.

Item Martín fijo de Pero Martiniz XX s.

Item Diago de Pangoa $\quad X X$ s.

Item Lope fijo de Johan Delgado XX s.

Item Ferrant Sanchiz de Merinteni XXs.

Item Johan fijo de Johan Delgado XX s.

Item la muger de Johan de los Huertos $\quad$ XX s.

Item Martín Martiniz notario XX s. 
Item Martín Ferrandiz de Pangoa XX s.

Item Johan Martiniz Manion XX s.

Item Johan Diaz de Billasuso XX s.

Item Martín Periz Garabalda XX s.

Item Johan Martiniz notario XXs.

Item Pedro Gonçalviz XX s.

Item Maria Diaz de Muga XX s.

Item Diago Alfonso XX s.

Item Pedro fijo de Pedro Lopiz XX s.

Item Martín de Burguera XX s.

Item Ferrant Sanchiz de Doroynno que es finado e en su logar Pedro su fijo XX s.

Item Johan de Araqa $\quad$ XX s.

Item Martín fijo de Gonçalo Sanchiz XX s.

Item Pedro Sanchiz sastre XX s.

Item Johan Lopiz fijo de Diago Lopiz XX s.

Item Johan Diaz de Doroynno XX s.

Item la muger de Pedro Ferrandiz de Salleo con sus fijos $\quad$ XX s.

Item Sancho fijo de Gomez Ferrandiz XX s.

Item Martín Sanchiz de Athahona con su fijo casado en casa XX s.

Item Johan de Amores $\quad$ X s.

Item Johan Loyna $\quad X \mathrm{~s}$.

Item Ferrant Gonçalviz $X$ s.

Item Pedro fijo de Pedro Sanchiz X $\quad$ s.

Item Pedro fijo de Johan Ferrandiz de Garixuri X s.

Item Martín fijo de Diago Lopiz Xs.

Item Maria Diaz X $\quad$ s.

Item Martín fijo de Johan de Hunxua Xs.

Item Johan Calvo X s.

Item Johan de Botoynno X $\mathrm{X}$.

Item Martín fijo de Johan Periz Xs.

Item Johan Sanchiz tondedor $\quad X \mathrm{~s}$.

Item Ferrando de Athahona X $\mathrm{X}$.

Item los fijos de Roy Sanchiz X $\quad$ X.

Item Ferrando de Berganço X $\mathrm{X}$.

Item Johan Lopiz cano Xs.

Item Pero Sanchiz de Luna X $\mathrm{s}$. 
Item Johan de Ysmellas $\quad X$ s.

Item Diago de Muga $\quad X$ s.

Item Johan fijo de Ferrando de Burguera Xs.

Item Martín rodero Xs.

Item Lope Ruyz Delgado X X.

Item Maria Yvannes Xs.

Item Theresa muger de Johan de Amores $\quad X s$.

Item Johan de la Cuesta $X$ s.

Item Johan Pelegrin el moço $\quad X s$.

Item Johan de la Builla con los fijos menores de Johan de la Cuesta X s.

Item Maria Lopiz X X.

Item Pedro de Biuxanda su yerno Xs.

Item Martín Pelegrin Xs.

Item Martín de Heras e Ferrando de Paredes X s.

Item Johan Gomez e Martín conejo X s.

Item Gonçalvo porqueriço $\quad \mathrm{V}^{\circ} \mathrm{s}$.

Item Johan de Metaute $\mathrm{V}^{\mathrm{o}} \mathrm{s}$.

Item Elvira Sanchiz biuda $\quad V^{\circ} \mathrm{s}$.

Item Johana de Doyuelo $\quad \mathrm{V}^{\circ} \mathrm{s}$.

Item Johan Sanchiz Poinça $\quad V^{\circ} s$.

Item Maria Sanchiz Biuda $\quad V^{\circ} s$.

Item Pedro Lopiz de Muga X s.

Item Ferrando de Doroynno Xs.

Item Theresa la de Johan de Garixuri e los fijos de Johan Ximeniz $\quad X$ s.

Item Johan Lopiz de Muga X $\mathrm{X}$.

Item Ferrando yerno de Johan de Amores $\quad X$ s.

Item los fijos de Johan Yvannes fijo de Pedro Yvannes X s.

Item Johan de Burguera $X \mathrm{X}$.

Item Johan Ferrandiz del Campo X $\mathrm{X}$.

Item Pedro trabuquero $\quad \mathrm{V}^{\circ} \mathrm{s}$.

Item Gonçalo Cardiel $\quad V^{\circ} \mathrm{s}$.

Item Ferrando de Hunxua $\quad V^{\circ} s$.

Item Johana de la Cuesta $\quad V^{\circ} \mathrm{s}$.

Item Johan de Tormantos $\quad X$ s.

Item Ferrando de la Cuesta $\quad V^{\circ} s$.

Item Ferrant Guiralde $\quad \mathrm{V}^{\circ} \mathrm{s}$.

Item Johan fijo de Johan Periz Adellino X s. 
Item Pedro Sanchiz notario con su madre X $\quad$ s.

Item Johan de Muga $X$ s.

Item Johan Periz de Johan Bueno X $\quad$ s.

Item Ferrando Yvannes de Dávalos $\quad X$ s.

Item Ferrando porqueriço Xs.

Item Johan fijo de Johan Periz clerigo Xs.

Item Sancho Desparça $X$ X.

Item Rodrigo e su hermano X s.

Item Martín de la Builla X X.

Item Johan fijo de Pedro Lopiz $\quad \mathrm{V}^{\circ} \mathrm{s}$.

Item Johan fijo de Johan de Garixuri $\quad \mathrm{V}^{\circ} \mathrm{s}$.

Item Johan el ferrero $X \mathrm{X}$.

Item Sancho Buquete $\quad V^{\circ} s$.

Item Johan Martiniz de Frango $V^{0} s$.

Estos de yuso contenidos dixieron que bivian en la dicha billa et los mantenian casi por amor de dios por que eran inpotentes et non pagavan cosa alguna.

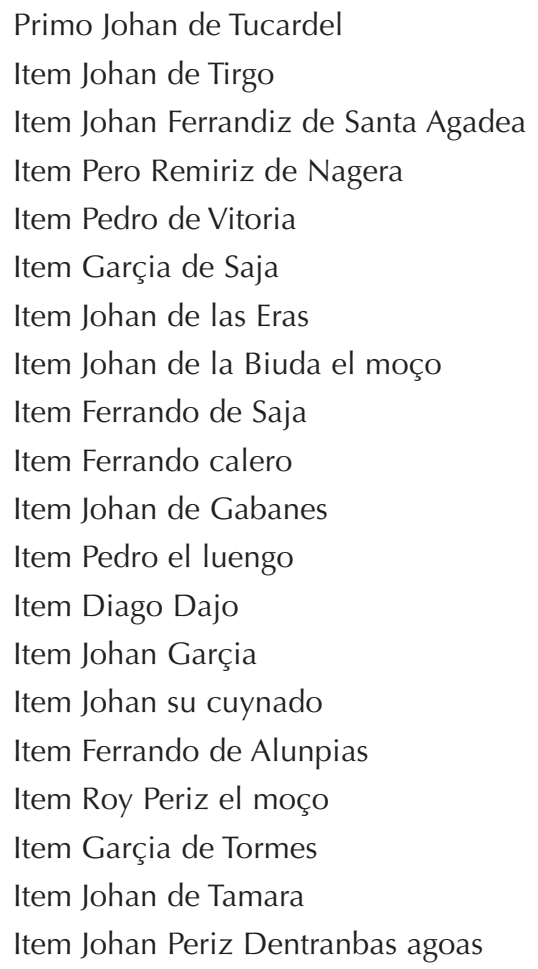


Davallos aldea de Sant Biçent

Et bien así nos los dichos comisarios reçebimos jura sobre la cruz et sanctos evangelios de Johan Remiriz de Dávalos.

Et sobre la dicha jura interrogados a los sobre dichos que avian jurado de la billa de Sant Biçent et bien así al dicho Johan Remiriz de Davallos quantos morada et casa mantenientes de clerigos et legos ay en la dicha aldea de Davallos dixieron sobre la dicha jura que ay los que se siguen:

Primo don Ferran Periz cura

$\mathrm{XXs}$.

Item don Pedro Yvannes fijo de Lope Yvannes

$X \mathrm{~s}$.

Item don Johan fijo de Sancho Periz

Xs.

Item don Ferrando fijo de Johan Periz

$X \mathrm{~s}$.

Item el dicho Johan Remiriz

$\mathrm{XX}$ s.

Item Martín Sanchiz Crespo

$\mathrm{XLS}$.

Item Maria Ferrandiz la de Roy Sanchiz

$X L s$.

Item Johan Ruyz fijo de Lope Ruyz

$X L s$.

Item Johana Lopiz

$X L s$.

Item Johan Sanchiz notario

$X L s$.

Item Pedro de Santa Maria

XX s.

Item Johan Ferrandiz de Sant Andres

$X X$ s.

Item Diago Ferrandiz fijo de Martin Ferrandiz

$X X$ s.

Item Sancho Ferrandiz

$X X$ s.

Item Yenego fijo de Martin Ferrandiz

$X X s$.

Item Martín Sanchiz de Laredo

$X X$ s.

Item Johan del Baille con su fija

$X X$ s.

Item Martín fijo de Johan Martiniz

$X X s$.

Item Ferrant Sanchiz

$X X$ s.

Item Sancho Periz

XX s.

Item Ferrando de Sant Andres

$X X s$.

Item Pedro Sanchiz de la Fuente grande

$X X$ s.

Item Johan de Billasuso

$X X$ s.

Item Johan Lopiz

$X X$ s.

Item Sancho Diaz

$X X$ s.

Item Lope fijo de Sancho Periz

$X X$ s.

Item Johan Blanquo con su fijo

$X X s$.

Item los fijos de Diago Lopiz

$X X$ s.

Item Lope de Billasuso

XXs. 
$\begin{array}{ll}\text { Item Johan de Borniello con sus entenadas } & \text { X s. } \\ \text { Item Lope Martiniz } & X \mathrm{~s} . \\ \text { Item Johan Sanchiz de la Caleja } & X \text { s. }\end{array}$

Item Martín Periz del Sel Xs. (tachado)

Item Lope Diaz X X.

Item Sancho del Baille X X.

Item María Ruyz X X.

Item María Martiniz X $\quad$ X.

Item Sancho Lopiz Xs.

Item Johan Sanchiz del Cortijo X $\quad$ S.

Item Gonzalo de Frango $X$ s.

Item Ferrando de Moraça X X.

Item Lope Ivannes X X.

Item Yenego de Hozana X $\mathrm{X}$.

Item Ferrando de Albiçio Xs.

Item Martin Yvannes X X.

Item María Ferrandiz de Sant Andres X X.

Item Johan de Toloynno del Baille X X.

Item Sancho Garçia de Cayzedo X X.

Item Pedro Yvannes Pacho $\quad V^{\circ} s$.

Item Sancho de Artaxona $\quad V^{\circ} s$.

Item Diago fijo de Pedro Yvannes X X.

Item Johana Ferrandiz $\quad V^{\circ} s$.

Item Martín Periz -tachado- Diago Fijo de Sancho Periz X s.

Item Pedro Ferrandiz fijo de Ferrant Yvannes X $\quad$ s.

Item Lope fijo de Pedro Sanchiz -tachado fijo- de la Fuente grande X s.

Item Johan Ruyz $\quad V^{\circ} s$.

Item Johan fijo de Sancho Periz $\quad V^{\circ} \mathrm{s}$.

Item Pedro el rodero $\quad \mathrm{V}^{\circ} \mathrm{s}$.

Item Johan carpentero X X.

Item Lope fijo de Johan Garçia X X.

Item Diago Sanchiz $\quad V^{0} s$.

Item estos de yuso contenidos sixieron que biven en el dicho logar et son inpotentes e non pagan cosa alguna.

Primo Pedro Gomiz de Frias

Item Pedro Ortiz 


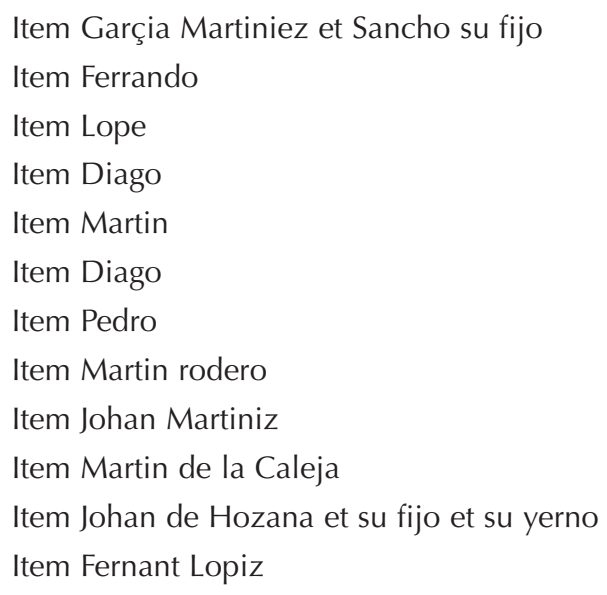

Picinna

Et bien assi reçebimos jura sobre la cruz et sanctos evangelios de Martin Remiriz de Piscina aldea de Sant Bicent

Et sobre la dicha jura interrogados los sobre dichos que avian jurado de Sant Bicent ensenble con el dicho Martin Remiriz quantos moradores casa mantenientes ay en la dicha aldea de Piscina dixieron que ay los que se siguen:

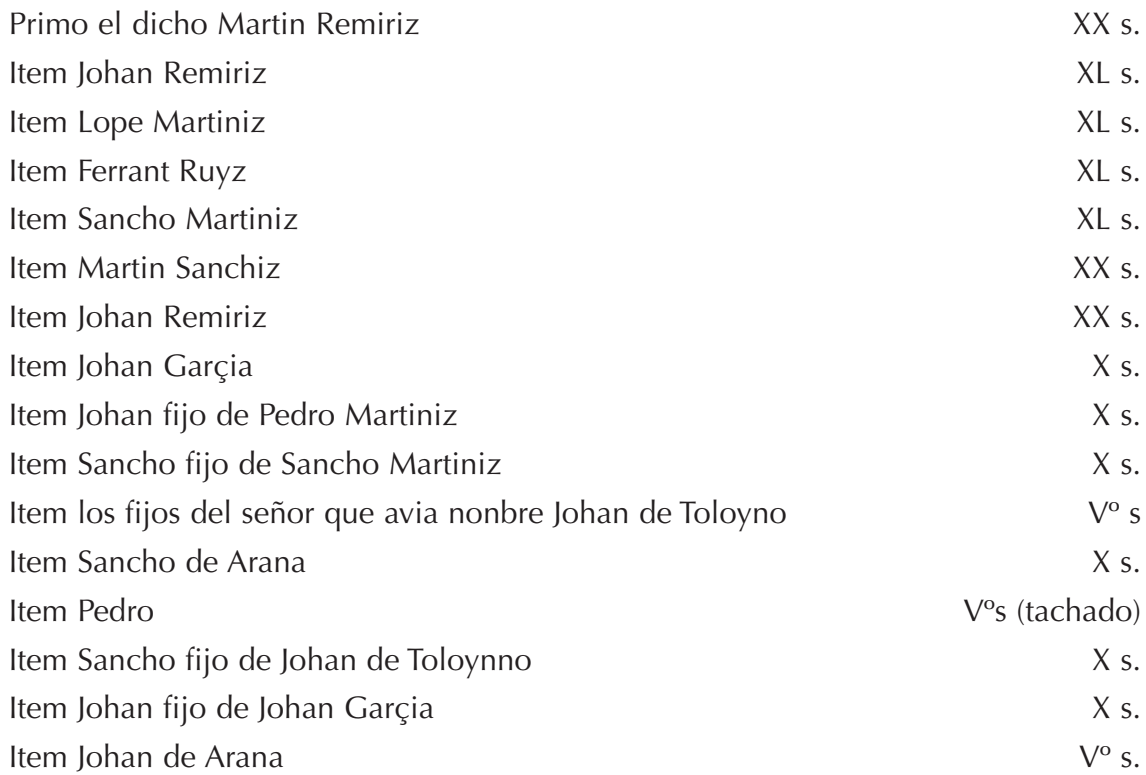


Item los de Yuso contenidos dixieron que bivian en el dicho logar et no an bienes algunos nin pagan cosa alguna

Primo Ximeno de la Bastida

Item Martin Periz ganadero

Item Pedro ganadero

\section{Orçales}

Et bien assi nos los dichos comisarios reçebimos jura sobre la cruz et santos evangelios toquandolos manualmente de Pedro de Orçales.

Et sobre la dicha jura interrogados los sobre dichos que avian jurado de la dicha billa de Sant Biçent et bien assi el dicho Pedro quantos moradores casa mantenientes ay en el dicho logar de Orçales dixieron que ay los que se siguen:

\begin{tabular}{|c|c|}
\hline Primo el dicho Pedro de Orçales con su madre & XXs. \\
\hline Item Johan de Toloynno & $\mathrm{XL} \mathrm{s.}$ \\
\hline Item Martin Yvannes & $X L s$. \\
\hline Item Diago Lopiz & $\mathrm{XL} \mathrm{s.}$ \\
\hline Item Johan Sanchiz & XXs. \\
\hline Item Ferrant Ruyz & X s. \\
\hline Item Martin Ruyz & X s. \\
\hline Item Pedro fijo de Ferrant Sanchiz & $\mathrm{V}^{\mathrm{o}} \mathrm{s}$. \\
\hline Item Pedro gorricho & $\mathrm{V}^{\mathrm{o}} \mathrm{s}$ \\
\hline Item Rodrigo fijo de Alfonsso & $\mathrm{V}^{\mathrm{o}} \mathrm{s}$ \\
\hline Item Johan fijo del Agoado & $X s$. \\
\hline Item la de Pedro fijo de Martin Yvannes & X s. \\
\hline
\end{tabular}

Riba aldea de Sant Bicent

Et bien assi nos los sobre dichos comisarios sobre la dicha jura interrogamos a los sobre dichos que avian jurado de Sant Bicent quantos moradores casa mantenientes ay en el dicho logar de Riba dixieron que ay los que se siguen:

Primo Johan Sanchiz Rojas

$\mathrm{XL} s$.

Item Johana Ferrandiz con sus fijos

$X L s$.

Item Ferrando fijo de Johan Sanchiz Rojas

$X s$.

Item Pedro fijo de Johan Ferrandiz

$\mathrm{Xs}$. 
$\begin{array}{lr}\text { Item Ferrando fijo de Johan Sanchiz page } & \mathrm{X} s . \\ \text { Item Johan su hermano } & \mathrm{X} s . \\ \text { Item Johan fijo de Johan Sanchiz } & \mathrm{V}^{0} \mathrm{~s} . \\ \text { Item Martin de Berganço } & \mathrm{V}^{0} \mathrm{~s} . \\ \text { Item Johan fijo de Martin Yvannes } & \mathrm{V}^{\circ} \mathrm{s} . \\ \text { Item Johan fijo de Johan de la Bastida } & \mathrm{V}^{0} \mathrm{~s} .\end{array}$

Interrogados que cargas an villa e aldeas dixieron que la billa de Sant Biçent et las aldeas de Piscina et Orçales pagan de Fossadera en cada un aynno C sueldos IXe dineros fuertes.

Item la aldea de Davalos paga en cada un aynno de fossadera a Roy Lopiz de Davalos.

Item que los de la aldea de Riba pagan en cada un ayno al abbat de Sant Salvador de Leyre eçeptado uno todos los otros moradores en el dicho logar cadaunno por el solar e casa donde bive cada $I I I^{\circ}$ rovos de trigo et cada sendos rovos de cevada que monta $X$ cafizes de trigo et II cafizes e medio de çevada poco mas o menos.

Item que la dicha villa de Sant Biçent et sus aldeas passan muy grandes afruentas con los frontaleros por quanto son situados en frontera et an bistas con XIle logares de castiIla et por cada debat o ruydo que acaezca de onbres et mugeres o de los de la billa les es necesario a yr a bistas con cadanno de los dichos logares donde se les seguesçen muy grandes espensas et daynnos por guardar sus terminos et los limites e confines de entre los regnos et guardar los drechos reales car si alguna sin razon o peyndra se faze por los de Castilla a estrangeros algunos en el territorio de Navarra por guardar la cura del sennor rey et los drechos reales et por non perder cosa alguna del territorio de Navarra eillos concejalmente se ponen e esfuerzan a defender et aun a seguescer a los tales tomadores et forçadores et con justiçia et a las bezes con su esfuerzo les fazen tornar de dentro de Castilla las tales cosas que seran tomadas non devidamente et retornarlas a sus dueynos et por seguescer estas cosas et guardar la cura et la onrra del regno passan muy grandes afruentos et se les seguescen muy grandes costas et por esta causa el sennor rey a quien dios perdone leyendo los grandes travajos et cargas que sostenian les fezo de gracia en los quoarteres que non pagasen por quarter sino la suma de XXBI florines e hun quarter et con todo avian pro que sostenerse por los grandes afruentos et cargas que sostienen et en Bitoria que an espendido por causa del pleito que lievan por Santa Maria de Toloyno et por la granja de Errameilluri porque son situados en el regno la suma de mil florines seguesçiendo el pleito en Roma e en otras partes por sostener el regno.

Interrogados si en la villa et aldeas an rentas provechos o revennas algunas dixieron que en la billa no ay rentas ni provechos algunos salvo que los montes quando cargan pazto suelen aver para mantener los puerquos que tienen para su provision et bien assi en las aldeas et que los de las aldeas se aprovechan algun tanto de la leyna que lievan a bender a Briones. 
Interrogados de que biven dixieron que la dicha billa et aldeas no an otra manera de bivir salvo que biben sobre la labranza de pan et bino e hun aynno con otro non cugen de pan para provision de la mitad del aynno et bino que cugen assaz para su provision et aun para bender enpero que del bino no se pueden aprovechar de que fue bedada la saqua del bino que non lo pueden bender sino a menoscabo de manera que casi no an provecho nin sostenimiento alguno.

Interrogados de la diminuyçion de la dicha billa et aldeas dixieron que destos XXXta aynnos aqua tanto por mortaldades como por algunos que son partidos a otras partes por las grandes cargas non podiendo bivir son diminuydos las dos partes que son IIC XL casas poco mas o menos de la dicha billa et de los que finquan valian mas $\mathrm{X}$ casas de las que son diminuydas que todas las que a presente quedan.

Item que en el dicho logar de Davallos son diminuydos senblanmente aca Lta casas. Item en Pizinna $\vee^{\circ}$ casas.

Item en Orçales III $^{\circ}$ casas.

Item en Riba II casas.

Et faillasse que la dicha billa de Sant Biçent con sus aldeas son taxsados por quarter CVo florines. 